\title{
Guidelines for Translators of Knowledge Text
}

Matthew Prattipati

\begin{abstract}
Each piece of translation is potential enough to convey the message of source text to its audience whether it is critical or creative. Translation transformed the lives of people in many nations. The history of translation declares that inscriptional inspirations to palm leaf practices, interpretations to interpretative communities, machine -human learning to soft talkie toddlers translation became an essential organ of human community. Historically speaking, the priorities and authorities catered labels of texts translations and translators. This paper focuses on the practicing translators and their specific needs.
\end{abstract}

Key words: Translation, translator, role of translator, practical needs and essential steps

\section{Introduction}

Knowledge text translation is different in its nature as any other texts which deal with the content or form. The translator of knowledge text should have some special characteristics along with acquaintance of that particular domain and preparation of translator for translating domain specific text is a must. There are some prerequisites for translator such as subject knowledge, writing ability in TL and good method of presenting the text. Translation as an activity has taken centre stage, particularly after globalization and there is need for localization or we could better term as "Glocal*". The Industry requirements for the present context would be:

Translation Today Vol.10, Issue-I, June 2016 
i) The specific discipline knowledge with some translating experience

ii) Competency in both the source language and the target language.

iii) A good comprehension of the source text.

iv) Critical and creative ability to search and select equivalents.

v) Critical and Creative ability to put the elements of a text together.

vi) Ability to access translation resources and also search online materials such as e-Dictionaries, thesauri etc.

It is a well-known fact that there is no substitute for practical experience of translation. To become a translator, there is no other option except doing practically the translation work. Translator should convey the original message of SL in TL without adding or deleting anything in the original message. The liberty of the translator depends upon assigning agency's guidelines given to the translator. It is also important to the translator to produce a reliable translation to the target readers. In the early days of translation it was believed that the translation is only the change of the language but not the message. Alexander Fraser Tytler (1791), Eugene A. Nida (1947) and others described in their writings, the characteristics of a good translator, here is the presentation of some of those for today's necessities. These are all not prescriptions but expectations. They are given below:

1. Excellent Knowledge of both the languages.

2. Excellent knowledge about two cultures

3. A good understanding and command over the subject matter which is being translated

4. Sensitive towards details of each aspect of the source text.

5. Readiness to read the available literature 
6. Support materials like dictionary, subject glossary, thesauri etc. to be available.

7. The specialized monolingual, bilingual and multi-lingual glossaries on the concerned subjects (such as legal, science, social and technical)

8. Linguistic ability to present thoughts in a convincing manner

9. Conceptual clarity and comprehensibility in the translated text.

10. Presenting natural, straightforward easy expression for a good readability

11. Providing acceptable and standard terms (literacy or non literary ) for proper understanding of the subject

12. Willingness to learn and refer any dictionary or encyclopedia or concerned subject experts for the purpose.

13. The quality and uniformity should be maintained in the entire text.

14. Avoid under translation or over translation

Besides the above characteristics, it is indispensable to note that to accomplish good work of translation. Mukherjee as cited in Kalyani (2001) suggested that "two more requirements for translator: first, the translator must be an avid reader and meaningmaker. By this statement he means that the translator must be a teacher, critic or editor so that the task undertaken could be done more satisfactorily. Second, the translator should be one who habitually writes in the TL. These are not rules prescribed for an ideal translator but are only suggestions to make a translator better in his work"

\section{What is the role of translator?}

Translation of any discipline whether it is Science, Computer Science, Engineering, Social Science and Humanities, it is 
very much significant that the translator has a vital role to play in the entire process of translating. There could be incalculable challenges while translating a text even for the expertise or professional translator. Every text is unique in its subject matter and in its own way, likewise translating each text is an exceptional experience for a translator and it defers from text to text, genre to genre and from discipline to discipline. It is important to know the role and responsibility bestowed upon the translator: (1) A translator is a meaning maker to the target language reader (2) The translator needs to encounter variety of issues not only to communicate a text from the source language to the target language, but also to do justice with uniformity of the expression, integrity in the subject matter.

\section{Preparation of the Translator}

Preparation is a must for translators besides his/her professionalism in their own domain. There is no doubt that the translators of any agency or publishing house are well established in the field of translation in their respective languages and subjects as well. This approach is quite different from the regular way of doing or translating a text. On many occasions, while translating a text from one language to the other, the confidence and competence of the translator in both languages comes into picture in mind than the intricacies involved in subject matter. Keeping in mind the users or target readers, the gravity of subject matter and researches on the knowledge text translation, prescribing this potential and systematic approach to avoid the untidiness in the translated texts and also to facilitate the translator in the best possible way. It is easy to implement and also makes the translator's task trouble-free, would be able to come up with substantial work. In the scientific and technical subjects or in any translation, the reliability and readability of the translation depends upon the understanding of the source text 
subject matter translating the target text with a natural flow of the language. The following concerns may give a vivid picture on the need for a different approach:

1. A good teacher of technical subject in both the languages (Source and Target language) may find difficulty in translating his/her own subject in a better communicative way.

2. The students with lack of English knowledge but having a desire to get into the scientific and technical education, to make their professional dream come true.

3. Bringing out the reader friendly translated texts of the Scientific or technical text in the academic environment.

4. Establishing relationship with the translated text, building the confidence among teachers, students on the subject.

5. Equipping the student-teacher community with a strong foundation and understanding of the subject.

6. The ultimate goal to build the nation as "Knowledge Power" from grass root to the higher stratum of the country.

In this regard, the following opinions on Scientific and Technical Translators and translation process are worth understanding.

On technical translation, Jody Byrne (2006) suggests that it is worth stating some of the important areas that need to be considered when translating technical texts:

1. We need to concentrate on the needs of the target audience for whom the translation is produced for and these are the judges of the translation work.

2. We need to understand what does the target audience expects.

3. We need to understand how technical communication works in the target language if we are to produce independent, 
autonomous texts that can compete with other texts produced in that language;

4. We need to remember that it is necessary to add, change or remove information as part of the translation process in order to achieve effective communication via a technical text (JODY BYRNE, 2006).

Apart from 1, 2, 3 point where it is a decision making area, the translators will have to consult experts to solve such issues as to add, change and remove any portion of the source text or for any untranslatability of the source text. And also, when a translator is not sure of any term or phrase, it is very much important on the part of the translator's to communicate with the assigned agency or authority.

Another perception of this issue was as follows: "Both Savory (1957) and Riess (1971) have written that a technical translator is concerned with content, the literary translator with form. Other writers have stated that a technical translation must be a literal, literary translation must be free"

(Newmark, P 1981). Looking at Mossop's categorization of the process of translating a technical subject is worthy in this present context. Mossop expresses the process of translation consisting of five tasks performed under three phases of translation production. The three phases are:

Phase 1: Pre-drafting

Phase 2: Drafting

Phase 3: Post-drafting

Task 1: Interpret the source text.

Task 2: Compose the translation. 
Task 3: Conduct the research needed for tasks 1 and 2.

Task 4: Check the draft translation for errors and correct if necessary.

Task 5: Decide the implications of the commission. (Jody Byrne 2006).

Keeping the earlier experiences, translating knowledge texts and assessing the translated works in various Indian Languages, discussing on this issue with the eminent scholars, prescribed the essential steps for the given task as given below for the practical needs:

Step 1: Reading the Source Text fully in its right sense.

Step 2: Write down the notes on where the flow of readability is breaking from the source text.

Step 3: Identifying the panic basket or real problems, which reduces the speed of the readability of source text.

Step 4: Collecting the available relevant resource material and studying it.

Step 5: Consulting the subject experts on this ground.

Step 6: Consulting experts (if necessary).

Step 7: Understanding the real problems.

Step 8: Second reading of the source text with good understanding.

Step 9: Taking down the details of words and phrases or even entire sentences which hamper the flow of writing.

Step 10: Classify the problems - what kinds of problems are occurring.

Step 11: Solve the problematic issues (Some issues, translator can solve, some problems Author can solve (if Alive), Subject experts can solve, some problems.

Step 12: Translators need to keep essential tools on hand for any translation work to give the best performance: 
a) Unabridged Monolingual Dictionary of Source Language is a must

b) Advanced Dictionary (of the Source Language)

c) Bilingual Dictionary (Source Language and Target Language)

d) A Comprehensive Encyclopedia of Source Language (Visual pictures if needed)

e) Reference Books, Electronic or Printed Dictionaries (in Source Language and if available, also in the Target Language)

f) Internet resources (online Dictionaries)

g) Discipline specific glossaries

h) A Good English language Thesaurus (if translator is dealing with English into any other language)

The translator must have update knowledge of the concerned subject's technical terminology. Translator is considered as the best reader of source text (so as to create readership for its knowledge text translation).

In recent times, online translation works has more in demand. If you are a translator of online assignments, you may not have much time to refer various online or offline resources and the above said steps may not have much importance due to time constrain. However, the translator may be equipped with essential available essential resources required for translating a text.

\section{What should not be translated?}

The following are some of the substance which translators should not translate:

Numbers : Most of the World's languages use Roman or Arabic numerals (1, 2, 3, 4,/ I, II, III, IV, V, VI, VII ), so it should not be 
translated. But in some languages, there has been a practice of using their own numerical, not the Roman. So the translator is suggested to take his/her own decision after knowing the practice.

Symbols: $、, \rightarrow, \Delta, \neq, \sum$; Formulas : r'2 /DL $\backslash$ n 7 i I /'max f -2 1 cos-edG $=-$ PmixDL $=$ F; Super script $:{ }^{\mathrm{nn}},{ }^{\mathrm{TM}}$; Names of the people : CV Raman, Benjamin Franklin; Names of the places etc : Mysuru,Vijayawada, Chennai, Pune and Kolkata

Acronyms should not be translated UNESCO IISc., IIM ect. and Names of the News Papers: The Hindu, Andhra Jyothi, Vijaya Karnataka and Praja Vani.

If the translators find any doubt or difficulty (during the translation activity) on any matter regarding what to translate and what not to translate), they (translators) should not hesitate to consult the senior scholars in that specific field.

\section{Selection of translation equivalents}

The Selection of equivalents in the target language is one of the most important factor in translation. The preference or choice of selection is very less for the translator when it is compared to Simultaneous/Oral translation. On many occasions, the translators run after transliteration which is not preferred. In this situation, translators are really in a quandary. It (transliteration) should be last option for them. The transliteration may be used if the translator is not able to find an equivalent term in the case of names of plants and animals, where genus and species are classified (for example: /chembaratti/in Malayalam and its scientific name is Hibiscus rosa-sinensis and Ginger**. Use of transliteration or translation equivalents depends upon the assigned agency or target readers. There may be a variety of equivalents on 
hand, but it is the responsibility of translator to provide appropriate equivalent in the target language. The translator should not go by postulation in his/her approach towards translation. The following may be just representation of such equivalents that creates a sticky situation to choose as well as to use. The translator must be aware of language nuances (Source or Target language) and the difference between Spoken verity and Written form. For example: స్కూల్ School. In Telugu language, irrespective of urban or suburban areas of Telangana and Andhra Pradesh, people use స్కూల్ School and in the rural areas బడి /baDi/ is in use and where as the Government records projects పాఠశాల/paaThaśaala/ for the same source concept School. Sometimes, for one term there may be many translation equivalents which need to be carefully opted for. The following examples gives clear picture

\section{English to Telugu}

\begin{tabular}{|c|c|c|}
\hline Source term & Transliteration & $\begin{array}{l}\text { Translation } \\
\text { equivalent } / \mathbf{s}\end{array}$ \\
\hline School & స్కూల్ / స్కూల్ & $\begin{array}{l}\text { పాఠశాల /paaThaśaala/ } \\
\text { బడి/baDi/ }\end{array}$ \\
\hline College & కాలేజీ, & కళాశాల /KaLaaśaala/, \\
\hline University & యూనివర్సిటీ & $\begin{array}{l}\text { విశ్వవిద్యాలయం } \\
\text { /Viśvavidyaalayam/ }\end{array}$ \\
\hline Computer & కంప్యూటర్/కంప్యూటరు & $\begin{array}{l}\text { సంగణకయంత్రం } \\
\text { /sangaNakayantram/ }\end{array}$ \\
\hline System & సిస్టమ్ & వ్యవస్థ/vyavastha/ \\
\hline Data & డేటా & $\begin{array}{l}\text { సమాచారం /samaacaaram/ } \\
\text { కార్యాలయం }\end{array}$ \\
\hline Office & ఆఫీసు & $\begin{array}{l}\text { /kaaryaalayam/ } \\
\text { పని /pani/, పదవి /padavi/, } \\
\text { బాధ్యత /baadhyata/ }\end{array}$ \\
\hline
\end{tabular}




\section{Culture and Ideology}

While translating a text, a translator may go through many experiences which may vary from time to time, context to context and translator to translator. It again depends upon the translator's competence, ideology, attitude etc. The following may give some insights into issues related to complexity of the subject matter of the text: A. Translating Knowledge Texts, especially, science, social science and humanities has its own way of expressing and organizing the content in a specific manner. It is a very common notion that in a translated text, there will always be some amount of loss in target text when it is compared with its Source Text. The authority over the subject and well acquainted with pragmatic nuances of the both languages is the real issue. It (the subject matter) may be complex and difficult to a translator who handles general article or books, but for a translator, having above characteristics may not find much difficulty to translate any subject. The subject matter should not be sacrificed for the sake of the other elements. Authors, translators and readers must treat both the translation and its source text equally.

While translating a text of social sciences, the translator may undergo the ethno-deviant pressures to produce on source text values into the target text. The translator tries to bring the likeliness in terms of linguistic and cultural difference. The translator should maintain a "creative balance" to bridge the gaps between the texts (source and target). The translator must understand the source text thoroughly and also read the available reference materials on that particular text. Translator is expected to maintain a creative balance between faithfulness of ST and naturalness of TL. For example, the concepts like "untouchable = 
అంటరాని /anTaraani/, హరిజన /harijana/ and Lower castes $=$ నిమ్నకులాలు /nimnakulaalu/, దిగువకులాలు/diguvakulaalu/ were overlapping each other (equivalents in target language -Telugu) in most of the cases. But, historically speaking, above three concepts occurred in different times and the context also defer. In this situation the translator need to take a decision and decide on the target language equivalents as mentioned above. This (harijana) concept was transliterated and the modern form was DALIT***, because of its semantic expansion in the present Indian context, the concept DALIT was used in a much broader sense and using the concept harijana considered as an offensive term. So the translator must have knowledge about historical developments and social changes which crop up in the society over a period of time. This kind of ethno-deviant pressures may hamper the rapidity of translation process. The translator must be much vigilant towards his/her endeavor to present an appropriate translation equivalent.

The concept and Context, if both are clear to the translator then it is very easy to process the text or to provide translation equivalents. For example: the term "Reception". There are four equivalents found suitable in different situations. The following are the equivalents: వివాహవిందు/vivaahavindu/ (marriagefeast), స్వాగత సభ /swaagata sabha/ (welcome-meeting), ఆహ్వానస్థలి /aahvaanasthali/ (invitingplace), రెసప్షన్/risepshan/ (transliteration form of the source concept). The translator must understand in a given context, which particular translation equivalent is healthy.

Translator should maintain the ideology of the source text; he/she should not put his/her own ideology in the translation. This is another critical issue to be addressed by the translator. The 
'ideology' of the source text may be retained. If the translator is not fit in the source text's ideology or the author's ideology better not venture into it. This is where the translator has to read and understand the source text in its right sense. And above all, target readers, assigned agency's guide lines determines the translation.

\section{Re-visiting the translation}

After completion of the translation task, it is very essential to study translation and check every detail of the source text whether properly communicated in the translated text or not. This will show the way to produce an authentic and good quality translation. Revisiting of the translated text helps the translator a) to detect the problems in the translated text, b) and to provide appropriate text or word. This process may appear to be odd to a professional translator because of her/his competence and experience in the field of translation. And also, due to monotonous and tedious exercise upon the source and target texts as well. The translated text should not hamper the temper of readability of target text, because of translator's hastiness and over confidence. Re-visiting of the complete text is another very important factor to be addressed. In re-visiting, the translator can also check: a) the basic concepts of the subject, b) style, c) consistency, d) central theme of the text. These are some of the important issues which has to be rethought after the first draft is ready. Therefore, the translators must understand the seriousness of the above said issues and take steps to correct these. The full text or the portion of the text translated by a single translator or two translators need to have a complete picture of the target text which facilitates the consistency in many respects. For example: If one word appears in more than one chapter and including the list of words in index, there is a possibility of giving various equivalents for one word by the same translator or different 
translators. It is necessary to have consistency in the text; to maintain the consistency or translator has to go through the text and correct such mistakes. While re-visiting, the translators can also find out, the translated text sounds "natural" to the target readers or not? If they come across any portion of the translated text or paragraph, they can re-write or translate those portions or paragraphs to be more natural to target readers or in target language.

Re-visiting brings the conformity of equivalence between the source text and target text by examining agreement of the source word meaning and target word in various levels. Such as word, phrase and beyond word level.

The Style of the translator also matters much when he/she presents the final version. In Telugu there are different varieties, which may be taken care of according to the situation.

1. Granthika (Classical variety )

2. Vyavahaarika(Modern variety )

3. Granthika to Vyavahaarika (Classical to Modern)

4. Vyavahaarika to Granthika (Modern to Classical)

It once again depends upon need, readers and text, time and assigned agency's demand.

\section{One can analyze the passage of source text and its translations (English to Telugu) Source text:}

There is also a danger, unhappily, that prospective students may ask advice from someone who knows about Linguistics, but who has at some time or other been offended by the subject. These people are quite numerous, in fact. The history of Linguistics, as of many new sciences, has been filled with clashes between 
disciplines, while the subject extended its field of study. A lot of this was due to exaggerated claims on behalf of Linguistics - claiming, for example, that one could not study the Classics without linguistic training, or that literary criticism was all wrong unless a thorough linguistic study of a text had first been made. But much was due to uncritical conservatism in people who worked in more wellestablished fields of study, and who did not like the critical examination which Linguistics was making of many cherished ideas. Nowadays, people pride themselves on being more enlightened and ecumenical in their views on the relationship of Linguistics to other subjects, but feelings can still be aroused. Moral: if one finds a person who reacts violently when asked what Linguistics is about, one should nod politely and ask someone else (David Crystal, 1969 pages4-5).

\section{Target text/Translation 1}

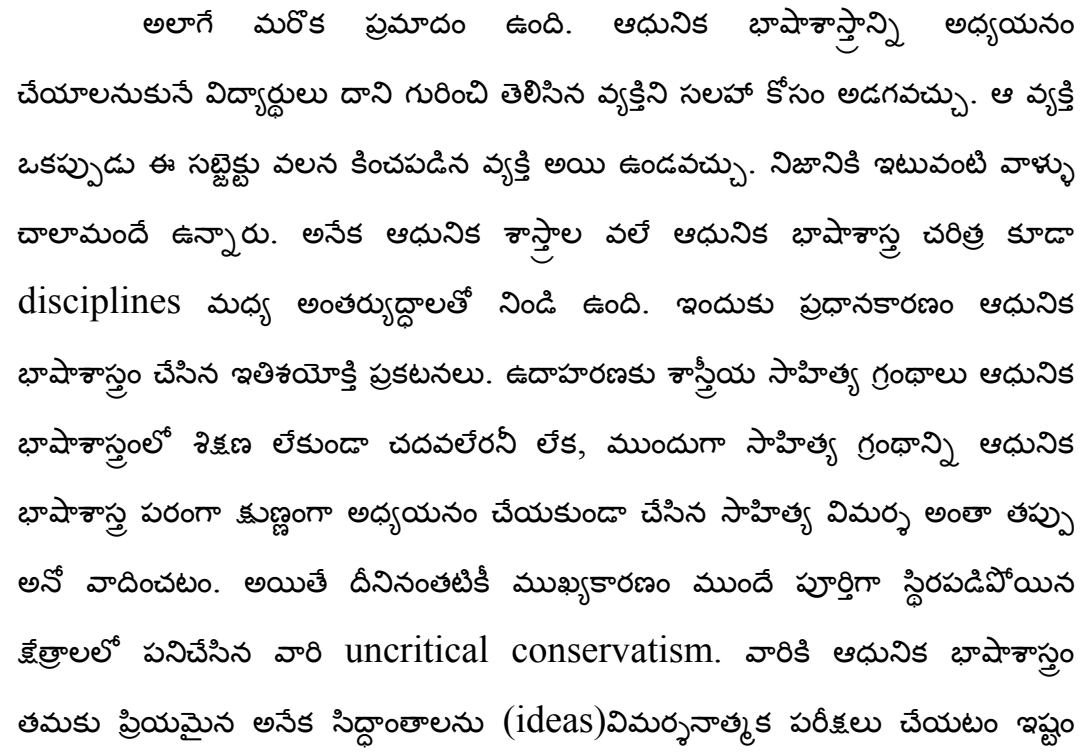


లేదు. ఈనాడు, చాలామంది ఆధునిక భాషాశాస్త్రానికి ఇతర subjects కూ గల సంబంధం పట్ల తమకు నిష్పక్షపాత, విశ్వజనీన అభిప్రాయాలు ఉన్నాయని గర్వపడుతుంటారు. అయినా ఉద్రేకాలు చెలరేగవచ్చు. నీతి: మీరు ఎవరికైనా ఆధునిక భాషాశాస్త్రం అంటే ఏమిటి అని అడిగితే ఆ వ్యక్తి చాలా ఉద్రేకంగా ప్రతిస్పందిస్తీ, మీరు మర్యాద పూర్వకంగా సరేనని చెప్పి ఇవతలకు వచ్చి మరొక వ్యక్తిని అడగాలి.

\section{Target text/Translation 2}

ఇక్కడొక ప్రమాదం కూడా ఉంది. భాషాశాస్త్రాన్ని అధ్యయనం చేయాలనుకునే విద్యార్థులు దాని గురించి తెలిసిన వ్యక్తిని సలహా కోరవచ్చు. ఆ వ్యక్తి ఒకప్పుడు ఈ సబ్జెక్టు వలన కించపడిన వ్యక్తి అయి ఉండవచ్చు. నిజానికి ఇటువంటి వాళ్ళు చాలామందే ఉన్నారు. అసేక ఆధునిక శాస్త్రాల వలనే భాషాశాస్త్ర చరిత్ర కూడా అధ్యయన శాఖల (disciplines) మధ్య ఘర్షణలతో నిండి ఉంది. ఇందుకు ప్రధానకారణం భాషాశాస్త్రం చేసిన ఇతిశయోక్తి ప్రకటనలు. ఉదాహరణకు ప్రాచీన సాహిత్య గ్రంథాలు భాషాశాస్త్రంలో శిక్షణ లేకుండా చదవలేరనీ, ఒక సాహిత్య గ్రంథాన్ని భాషాశాస్త్ర పరమైన సమగ్ర అధ్యయనం చేయకుండా చేసిన సాహిత్య విమర్శ అంతా తప్పు అనీ వాదించటం. అయితే దీనిసంతటికీ ముఖ్యకారణం అప్పటికే బాగా పనిచేసిన వారి (uncritical conservatism.) సంప్రదాయవాదం. భాషాశాస్త్రం తమకు ప్రియమైన అనేక సిద్ధాంతాలపై (ideas) విమర్శనాత్మక పరీక్షలు చేయటం వారికి ఇష్టం లేదు. ఈనాడు, చాలామంది భాషాశాస్త్రానికి ఇతర subjects కూ గల సంబంధం పట్ల తమకు వివేకవంతమైన విశ్వజనీన అభిప్రాయాలు ఉన్నాయని గర్వపడుతుంటారు. అయినా ఉద్రేకాలు చెలరేగవచ్చు. నీతి: మీరు ఎవరినైనా భాషాశాస్త్రం అంటే ఏమిటి అని అడిగితే ఆ వ్యక్తి చాలా ఉద్రేకంగా స్పందిస్తే, మీరు మర్యాద పూర్వకంగా సరేనని చెప్పి ఇవతలకు వచ్చి మరొక వ్యక్తిని అడగాలి. 


\section{Analysis of translations:}

If we see the term 'Linguistics', the Translation-1 provides ఆధునిక భాషాశాస్త్రం/aadhunika bhaashaa saastram/ as an equivalent and Translation-2 gives భాషాశాస్త్రం (bhaashaa saastram) for the source term.

The term 'Linguistics' in the source text was used in its broader sense, but the Translation1 used ఆధునిక భాషాశాస్త్రం/aadhunika bhaashaa saastram/ as an equivalent. By using the concept "Modern" /aadhunika/as a prefix to 'bhaashaa saastram', confines the scope of source term only to the Modern Linguistics. And, where as in Translation 2 used word భాషాశాస్త్రం/bhaashaa saastram/ which fits appropriately to the term' Linguistics' in the target language. The equivalence plays vital role in translation, especially in terms of accuracy in meaning and relevance in the given context. Translators should understand the source word meaning in its given context and produce the same as exactly as possible into target language word (Telugu) A word/term/phrase meaning depends upon not only on its context but also the place and time. When we see the term 'Subject' in Translation 1 and 2 both the translators are borrowed the term in its transliterated form and nativized as 'సబ్జెక్టు'/sabjekTu/.

And, the plural form of term 'Subject' also appears in the source text as 'Subjects', the translators of 1 and 2 hold on to the source word and retained as it is. In other term like 'Disciplines', translator of Translation 1 retained the source word as it is 'disciplines' and the translator of Translation 2 translated the word as అధ్యయన శాఖలు (disciplines) and also given the source word within the brackets. The word like 'Clashes', both the translators (Translation 1 and 2) translated as అంతర్యుద్ధాలు/antaryuddhaalu/ in 
Translation 1 and ఘర్పణలు/gharshaNalu/ in Translation 2.

The complex word 'antaryuddhaalu' literally means 'internal wars' or 'internal clashes' in the target language, and the translation 2 presents appropriate equivalent 'gharshaNalu' for the source word 'Clashes'. The concept 'conservatism' is retained by the translator 1 as 'conservatism' and translator 2 translated as సంప్రదాయవాదం/sampradaayavaadam/ and also the source concept 'conservatism' given within the brackets.

\section{Syntax level:}

a There is also a danger

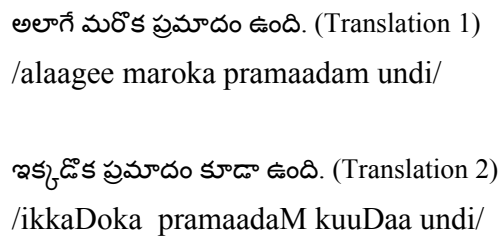

The above sentence translation, the translation1 used the words అలాగే మరొక/alaagee maroka/ which literally means 'likewise another' and translation 2 gives হక్కడొక/ikkaDoka/ which means 'here one'. While translating this sentence, Translator1 and 2, deviated a little from the source text. The appropriate translation for the above source sentence would be అక్కడొక ప్రమాదం కూడా ఉంది.

b These people are quite numerous, in fact.

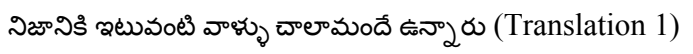


నిజానికి ఇటువంటి వాళ్ళు చాలామందే ఉన్నారు (Translation 2)

/nijaaniki iTuvanTi vaaLLu caalaamandee unnaaaru/

Translator 1 and 2 translated in the same way which conveys the source sentence meaning.

c Moral: if one finds a person who reacts violently when asked what Linguistics is about, one should nod politely and ask someone else.

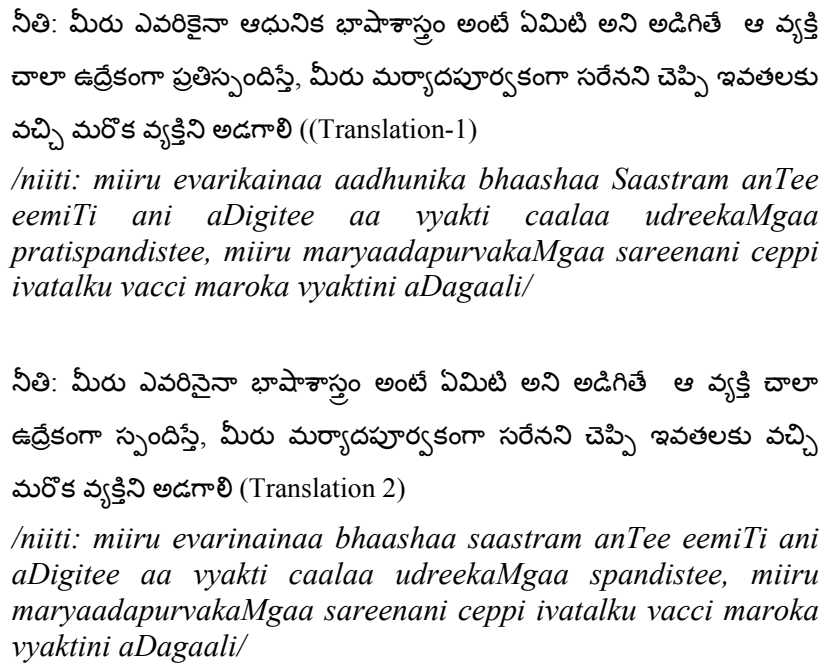

When we compare the complex sentence of the source text with above two of its translations (Translation - 1 and Translation - 2), Translation-1 ఎవరికైనా /evarikainaa/ (which means' for anybody') not be suitable in this context but Translation - 2 used appropriate equivalent as ఎవరినైనా /evarinainaa/ ('to anyone'). The selection of appropriate equivalence plays major role in translation. The source word 'react' has /pratispandistee/ ప్రతిస్పందిస్తే, in translation1 and /spandistee/ స్పందిస్తే used in translation-2. These two equivalents are not so different but 
translation- 2 would be better. Translator 1 and 2 followed the structure of Telugu syntax. In the above two translations readability and communicative effect are good but Translation-2 is more accurate than Translation-1.

\section{Conclusion:}

The basic idea of this entire paper is to promote translation as a regular household activity and to produce quality translation. This also equally aims at the working class to accelerate their proficiency and finally to the business class to understand practical issues involved in this very act of translation.

\section{Notes:}

1. *Glocal: Reflecting or characterized by both local and global considerations: in the Web 2.0 era, every public institution has already been transformed into a glocal enterprise.

2. **Ginger (Zingiber officinale Rosc.) Zingiber is derived from the Greek "zingiber" which is originated from Prakrit "singabera", from Sanskrit srngaveram, from srngam "horn" + vera- "body" and meaning is 'shaped like a horn'. And, its roots were in Malayalam for the spice, inchi-ver, from inchi "root."

3. ***Dalit: "The word Dalit is derived from Sanskrit language and, etymologically, it means "ground", "suppressed", "crushed" or "broken to pieces". Hence, by connotation, the term Dalit is used as an adjective or noun to describe the people or communities that have remained down-trodden or at the margins of society throughout India's long social history (Shivanand, 2014)." 


\section{REFERENCES}

Tytler, Alexander Fraser. 1791. Essay on the Principles of Translation, J.M.DENT-\&CO: London.

Benjamin, Walter. 2004. The Task of the Translator in Venuti, Lawrence, ed. The Translation Studies Reader, Routledge: London.

Gouadec, Daniel. 2007. Translation as a Profession, John Benjamins Publishing: Amsterdam.

Crystal, David. 1969. What is Linguistics? Edward Arnold: 41 Maddox Street, London WI.

Dadawala, Darshini. 2016. Dalit Literature - Concept, Origin and Features, International Journal of English Language, Literature and Humanities, Volume IV Issue II. pages 11-14., www.ijellh.com.

Nida, Eugene A. 1947. Bible Translating, United Bible Societies: London.

----------(1964) Towards a Science of Translating, Leiden: E. J. Brill.

----------and Taber, Charles. (1969) The Theory and Practice of Translation, Leiden: E. J. Brill.

Samuelsson-Brown, Geoffrey.1993. A Practical Guide for Translators, Multilingual Matters : Bristol.

Byrne,Jody. 2006. Technical Translation Usability Strategies for Translating Technical Documentation, Springer: The Netherlands.

Kalyani, P.K. 2001. Translation Studies, Creative Books: New Delhi.

Aranda, Lucía V. 2007. Handbook of Spanish-English Translation, University Press of America: Maryland.

Hann, Michael. 2004. A Basis for Scientific and Engineering Translation, John Benjamin Publishing Company: Amsterdam

Sofer, Morry. 1996. The Translators Handbook, Schreiber Publishing: Rockville.

Newmark, Peter. 1981. Approaches to Translation, Oxford: Pergamon Press. 
1988. A Textbook of Translation, New Jersey: Prentice Hall.

Pinchuck, Isadore. 1977. Scientific and Technical translation, André Deutsch: London.

Krishmamurthy, Ramesh. 2001. Indian Tradition in Baker, Mona. (2001) Ed. Routledge Encyclopedia of Translation Studies, Routledge: London.

Reiss, Katharina. 1971. Possibilities and Limitations of Translation Criticism: Categories and Criteria for a Proper Assessment of Translations, München: Hueber.

Savory, Theodore. 1957. The Art of Translation. London: Jonathan Cape.

Hunashimarad, Shivanand I. 2014. Origin and Development of Dalit Community, International Journal Of Innovative Research \& Development, Vol 3 Issue 5 Page 84-85., www.ijird.com.

Acknowledgements: Author is very much thankful to Prof. V. Saratchandran Nair, Project Director of NTM for his valuable suggestions on the earlier version of this article and also NTM Staff. 